\title{
Hospital de día psiquiátrico para niños y adolescentes ${ }^{1}$
}

\section{Introducción:}

Este trabajo es el resultado de diversas reuniones de profesionales pertenecientes a dispositivos específicos para un abordaje clínico de la patología mental grave en niños y adolescentes (se incluye lista al final) convocados y organizados por la Sección de Salud Mental Infanto-Juvenil de la A.E.N.

De la situación actual en las distintas comunidades autónomas en relación a los dispositivos intermedios o de hospitalización parcial podríamos concluir:

Los actuales dispositivos clínicos para el abordaje de la patología mental grave en infancia y adolescencia que existen en las distintas comunidades autónomas parten en muchos casos de diferentes formas de organización y de ubicación, perteneciendo además a diferentes organismos, siendo sus características las del hospital del día y lo común la definición de terapia institucional.

En todos estos dispositivos, y para los niños y adolescentes en edad escolar, el tiempo de permanencia diario en los mismos está en función de sí es posible la integración escolar o no: tivo.

* Niños integrados en sus colegios correspondientes: tiempo parcial en el disposi-

* Tratamientos integrados con educación: tiempo total en el dispositivo.

En cualquier caso lo que se ve como necesario, es que en cualquier vía que se elija o se tenga, a de ser imprescindible la coordinación con educación y la integración funcional entre ambos (sino está el peligro de un funcionamiento en redes paralelas).

Estos dispositivos son:

a) Unidades de crisis, en estructura sanitaria con tiempo breve de estancia, en régimen de día (máximo 29 días en algún dispositivo)., con objetivos de contención, evitar o acortar la hospitalización, movilizan sintomatología de bloqueo masivo (fobia escolar), como paso a un proceso terapéutico ambulatorio o a una hospitalización de día a tiempo prolongado.

Este dispositivo está diseñado para latentes y adolescentes.

b) Centros específicos: dependientes de Educación, y planteado como escuelas especiales con personal de Salud Mental (psiquíatra, psicólogo), que cubre necesidades sanitarias y educativas. Con un tiempo medio de estancia de varios años, con financiación mixta (Enseñanza, Sanidad y familias).

1 Trabajo presentado en el último congreso de la AEN, celebrado en Santiago de Compostela, en Junio de 2000

Rev. Asoc. Esp. Neuropsiq., 2001, vol XXI, n.0 77, pp. 115-124 
c) Hospitales de Día a tiempo parcial, con objetivos psicoterapéuticos que incorporan diferentes abordajes integrales para niños con trastornos emocionales severos, pero que están integrados en sus centros escolares correspondientes.

d) Abordaje en régimen de Hospital de Día a tiempo completo. Engloban diferentes técnicas con el objetivo de lograr la progresión en el desarrollo emocional y cognitivo. Aborda patología emocional severa intentando en la medida de lo posible no separar al niño o adolescente de su medio escolar, social y familiar, propiciando su relación con el mismo.

e) Colegios de educación especial: Que entrañan cambios estructurales que permiten convertir el centro permanente pedagógico y rehabilitador en una dimensión de comunidad terapéutica.

Se plantean 2 modelos, uno más centrado en lo terapéutico y otro en la rehabilitación psicosocial.

\section{H.D.P.N.A. Marco general}

Es importante destacar en primer lugar que el hospital de día psiquiátrico para niños y adolescentes debería estar adscrito a la red de asistencia de Salud Mental de niños y adolescentes, dentro de la red asistencial de Salud Mental general siendo necesaria la existencia además de otros dispositivos que asegurasen las prestaciones básicas para estas edades como son:

1 - Equipo básico ambulatorio que atendería:

1.1 - Demanda asistencial existente.

1.2- Relación con Atención Primaria y Actividad Preventivas

1.4- Tareas de formación e investigación.

2 - Nivel de hospitalización:

2.1- Urgencias, atendidas por lo general por el dispositivo de psiquiatría general, durante las 24 horas; importante la coordinación con los dispositivos ambulatorios.

2.2- Interconsulta y/o psiquiatría- psicología de enlace.

2.3- Hospitalización total de niños y adolescente por trastorno mental. Se estipula para una población de 250.000 habitantes, 2-4 camas para niños, 4-6 camas para adolescentes. Este dispositivo puede estar ubicado en la planta pediátrica (aunque dependiendo de psiquiatría) o de psiquiatría , pero en ambos casos separado de la misma y debidamente acondicionada, o constituir una unidad de agudos independiente (como pasa en grandes poblaciones).

2.4- Dispositivo para patología con importante trastorno de conducta con tendencia a la psicopatía :

* Centro terapéutico y rehabilitador de media estancia, con intervención mixta de Sanidad, Educación y Bienestar Social, para trastornos mentales cronificados, con condiciones sociales y familiares muy desfavorecidas o difíciles, cuyas características habría que definir claramente. 
INFORMES

\section{Definición general}

El Hospital de día para niños y adolescentes es un dispositivo asistencial para el tratamiento institucional intensivo, en un medio terapéutico especifico, de los trastornos mentales severos que aparezcan en estos periodos de la vida y que tienen en común el provocar una perdida en sus posibilidades evolutivas y de vida autónoma, así como serias dificultades en la vida relacional social y familiar, estando asimismo seriamente alteradas sus capacidades académicas o profesionales.

Debido a la gran disparidad de las edades, se hace necesario especificar claramente tres espacios diferenciados dependiendo de la edad:

-De 2 a 6-7 años.

-De 6-7 años a 12.

-De 12-13 a 17 años inclusive.

El tiempo de permanencia semanal en el dispositivo, es variable, estando en función de la posibilidad o no de integración en los centros escolares de la red educativa de cada área geográfica o de los centros prelaborales.

El HDP de niños y adolescentes se diferencia de otros dispositivos que no tengan el claro carácter terapéutico como son:

- Centros de día de carácter psicoeducativo o rehabilitador, con objetivos más específicos de adaptación sociofamiliar y/o laboral.

- Centro o aulas de autistas (y demás psicosis) fundamentalmente de carácter educativo.

- Talleres "del fondo de garantía social".

- Equipos de Atención Temprana, con abordajes de estimulación precoz para niños con diferentes discapacidades

- Centros o programas para tratamientos ambulatorios, que aunque de carácter terapéutico, no imparten tratamiento intensivo, dentro de la concepción de la terapia institucional.

- Las Unidades de Crisis para patología mental severa, que por su breve tiempo de estancia, no pueden considerarse como un dispositivo de hospital de día, siendo necesario en muchos casos ambos recursos.

\section{Objetivos terapéuticos}

1- El objetivo fundamental seria el posibilitar el cambio emocional y cognitivo de forma estable, que permita una mejor adaptación a la realidad, así como una mejora de las competencias relacionales, permitiendo una reincorporación a la vida en la comunidad, donde podrán continuar el tratamiento ambulatorio oportuno.

El tiempo total en el dispositivo dependerá de la posibilidad de cambio y no solo sintomatológico y debería ser hasta: 
- En las evoluciones positivas la posibilidad de un tratamiento más adecuado en régimen ambulatorio.

- En el establecimiento de cronicidad más definitiva, obligando a pasar a dispositivos más adecuados (Centro psicoeducativo o rehabilitador, talleres de garantía social...)

2- Trabajo con la familia, ayudando a contener y elaborara las ansiedades y ambivalencias generadas por la enfermedad y actuando sobre los factores familiares influyentes en la misma.

3- Trabajo con el medio escolar (en niños y adolescentes integrados escolarmente) y social, favoreciendo esta integración y actuando sobre los factores que en ambos ámbitos puedan estar influyendo o agravando el cuadro clínico.

\section{Indicaciones y perfil del paciente}

\section{Indicaciones clínicas}

Población entre 2 y 17 años (ambos inclusive)que presente cuadros psicóticos u otras alteraciones severas que conlleven un trastorno de la organización del yo y de la relación del niño/ adolescente con su mundo circundante y que impidan su adaptación al medio familiar, social, escolar y /o profesional. Se incluyen:

-Trastornos generalizados del desarrollo.

- Esquizofrenia, Trastorno Esquizotípico.

-Trastornos del Humor.

- Trastornos neuróticos severos.

- Trastornos de personalidad.

- Disarmonías evolutivas severas.

- Trastornos graves de la alimentación.

- Trastornos de la comunicación, etc.

Inicialmente si son admitidos, aunque con ciertas condiciones:

- Las patologías con trastornos de conducta graves (depende de la capacidad de control del grupo).

- Niños/ adolescentes con bajo nivel intelectual y con trastorno mental (depende del nivel de su capacidad de elaboración mental).

- Trastorno mental grave injertado en cuadro psicoorgánico (así mismo dependera de la capacidad de elaboración mental).

Contraindicaciones

- Conducta psicopática estructurada.

- Consumo de tóxicos como primer diagnostico o con persistencia de un consumo de riesgo.

- Conductas suicidas que no puedan ser controladas cuando el niño o adolescente estén fuera del dispositivo. 
INFORMES

- Trastornos que impidan la capacidad de elaboración mental como son:

* Retraso mental (en grados moderado o severo).

* Trastornos psicoorganicos.

* Déficit cognitivos definitivamente establecidos, de diversas etiologías.

\section{Actividad terapéutica}

Bases del tratamiento

La intervención terapéutica está basada en que todo niño/ adolescente con trastorno mental grave, tiene una vida psíquica propia y una posibilidad de vida relacional con el entorno, por muy deteriorado que esté.

Por una parte el equipo terapéutico a de poder vincularse al niño/ adolescente para contener sus angustias, favoreciendo los procesos de pensamiento.

Por otra la vida relacional a de posibilitar la expresión de ansiedades, afectos, conflictos intrapersonales y relacionales..., a través de un ámbito contenedor, firme y estable, que garantice una continuidad de cuidados, para el logro de un cambio que vaya más allá de la simple desaparición o disminución de síntomas concretos.

Así mismo la terapia institucional a de favorecer la relación entre los niños o adolescentes, siendo esto uno de los factores favorecedores del cambio psíquico.

\section{Plan terapéutico}

Con los niños/ adolescentes

- Actividades libres (expresión de la vida emocional y sus conflictos).

- Actividades encaminadas a establecer un vinculo relacional.

- Actividades de psicoterapia: reunión grupal (priorizar la utilización del lenguaje), psicodrama, psicoterapia dinámica de grupo.

- Psicomotricidad.

- Logopedia; trabajo psicopedagógico.

- Funciones de sociabilidad.

-Expresión plástica y corporal.

-Actividades de taller (trabajo con barro, madera, pintura...).

-Tratamiento psicofarmacológico.

Con las familias

-Grupo de orientación y atención de padres.

-Intervenciones familiares individuales.

Con el nivel escolar (para niños y adolescentes integrados en centros educativos)

-Coordinación y articulación en cada caso con los profesionales de educación.

-Intervención concreta escolar: reuniones periódicas con el profesorado correspondiente para comprensión emocional del niño/ adolescente, contención de la ansiedad ge- 
INFORMES

nerada, búsqueda de soluciones más idóneas para favorecer la integración.

Coordinación con dispositivos de Bienestar Social

- Búsqueda alternativas periodo vacacional; integración en actividades de tiempo libre, búsqueda subvenciones...

Coordinador con Justicia y Trabajo y organizaciones tutelares, favoreciendo la continuidad de cuidados en estas ultimas.

\section{Organización}

\section{Ubicación física}

Ha de estar integrado en la red sanitaria, pero debido a que en estos momentos la financiación no es uniforme (INSALUD- Sanidad, Educación para los Centros de día con orientación terapéutica, Bienestar Social para dispositivos de atención temprana y/o financiación privada- familiar), todos los dispositivos considerados bajo el concepto de hospital de día (o centro terapéutico de día) han de estar coordinados dentro de la red asistencial.

\section{Capacidad asistencial}

Para cada grupo de edad la capacidad puede oscilar, admitiéndose un máximo de 15 niños o adolescentes en cada grupo.

\section{Recursos humanos}

Tanto el nivel madurativo y emocional, como las manifestaciones psicopatológicas, varían en las diferentes edades, lo que condiciona necesidades también diferentes en los recursos humanos para abordarlas.

Partiendo en todos los casos de la necesidad de equipos multidisciplinares, donde se utilicen técnicas psicoterapéuticas para los trastornos emocionales, junto con trabajo pedagógico necesario para el desarrollo cognitivo, potenciando las capacidades del Yo (lo que a su vez es necesario así mismo para el desarrollo emocional), en cada dispositivo se requieren:

- Hospital de día para niños de 2 a 6 años (Para 15 niños)

Personal a tiempo completo:

- 3 Monitores/as (cuyo perfil profesional puede encajar en auxiliares de clínica, técnicos de jardín de infancia...).

- 1 maestro/a especializado en Pedagogía terapéutica.

Personal a tiempo parcial

- 1 psiquiatra de niños y adolescentes.

- 1 psicólogo clínico.

- 1 logopeda.

- 1 psicomotricista. 


\section{INFORMES}

- 1 Enfermero/ a psiquiátrico.

- Hospital de día para niños de 6-7 a 12 años (para 15 niños)

Personal a tiempo completo

- 2 monitores/ as (del mismo perfil profesional que los anteriores)

- 1 - 2 maestros especializados en Pedagogía terapéutica.

- 1 terapeuta ocupacional.

Personal a tiempo parcial

- 1 psiquiatra de niños y adolescentes.

- 1 psicólogo clínico.

- 1 logopeda.

- 1 psicomotricista.

- 1 enfermero/ a psiquiátrico.

- Hospital de día para chicos de 12 a 17 años (Para 15 chicos/ as)

Personal a tiempo completo

- 2 monitores (mismo perfil).

- 1 terapeuta ocupacional.

- 1 enfermero/ a psiquiátrico.

Personal a tiempo parcial

- 1 psiquiatra de niños y adolescentes.

- 2 psicólogos clínicos.

- 1 subunidad pedagógica con profesores, que dada la diversificación de materias que se deben abordar, puedan cubrir las necesidades en este terreno de chicos/as tanto integrados en sus centros escolares correspondientes, como de los que permanecen a tiempo completo en el dispositivo.

Para los tres grupos de edad, es necesario contar con que los distintos psicoterapeutas puedan ofrecer por su formación diferentes técnicas psicoterapéuticas: individuales, grupales, de orientación dinámica, de orientación cognitiva- conductual...

Así mismo cada dispositivo a de contar con un auxiliar administrativo, que puede ser propio o compartido con los dispositivos de la red de Salud Mental.

También deberá contar con un Trabajador Social compartido con los que estén ubicados en la Red de Salud Mental.

\section{Requerimiento mínimos de espacio}

Debería de contar al menos con:

- Sala de espera, administración, archivo clínico, despachos para facultativos, sala 
de reunión y biblioteca; así mismo área de enfermería, con botiquín y material sanitario y camilla (todos estos espacios serán compartidos en caso de que esté ubicado el HDP dentro de otro dispositivo asistencial).

- Área de observación y relajación para situaciones de agitaciones y trastornos de conducta, reacciones adversas...

- Sala de grupo, o espacio polivalente.

- Sala o taller ocupacional.

- Sala de grupo para trabajo más cognitivo (logopedia, psicopedagogía...).

- Espacio abierto, de ocio (patio o similar...).

- Cocina u ofis en caso de que la comida se elabore en el centro.

- W.C.

Todo ello con espacio suficientemente amplio.(En total 100 metros aproximadamente).

Recursos materiales diagnósticos y terapéuticos

- Batería de test apropiada para cada edad.

- Material lúdico suficiente.

- Material psicopedagógico suficiente y amoldado a cada edad.

Es necesario para completar este documento elaborar sistemas de evaluación de estos dispositivos.

Asimismo es importante elaborar protocolos de derivación a este dispositivo, para niños pequeños, ya que suelen ser diagnosticados muy tarde, siendo necesario además diferenciarlos de los casos atendidos más específicamente en dispositivos de Atención (estimulación) Temprana. 
INFORMES

Participantes en el grupo de trabajo sobre Hospital de Día Psiquiátrico para niños y adolescentes

\begin{tabular}{|l|l|}
\hline JAIME CLARET & CARME TELLO CASANY \\
Psiquiatra & C. S. M.I.J. LLEIDA \\
Hospital de Día S. Juan de Dios-Barcelona & Bisbe Torres, 2 - 25002 - LLEIDA \\
Telef. 93 2804000 - 4156800 & Tel. 973 267271 - Fax: 973 282710 \\
& E-mail: csmijll @iname.com \\
\hline MERCE GIBERT & Ma JOSEFA IRIBARREN CIA \\
Psiquiatra & Psicóloga \\
Hospital de Día de Gavá & Hospital de Día Infanto Juvenil \\
Telef. 93 6384364 & «Natividad Zubieta» \\
& C/ S. Cristóbal, sn. - PAMPLONA \\
& Tel. 948 13 66 66 \\
\hline RAMON MARTI & ANTONIO QUETGLAS PONT \\
Centro Alba - BARCELONA & Psiquiatra \\
Telef. 93 4183676-932118328-972212956 & Hospital de Día Infanto Juvenil \\
& «Natividad Zubieta» \\
& C/ S. Cristóbal, sn. - PAMPLONA \\
Tel. 948 13 66 66 \\
\hline INGRID THELEN & Mª TERESA PI ORDOÑEZ \\
D. Enfermería & CSMIJ - Girona \\
Hospital de Día de Adolescentes & C/ Antoni Varés i Martinell, 40 \\
Hosp. Sta. Caterina - GIRONA & 17007 -GIRONA \\
Telef. 972 - 182500 - Ext. 590 & Tel. 972 224749 - Fax: 972 226204 \\
\hline ANDRES GARCIA SISO & MARGARITA IBAÑEZ FANES \\
C. S. M.I.J. Tarragona & Servicio de Psiquiatría. \\
Instituto Pere Mata & Hospital S. Juan de Dios. \\
C/ Instituto Pere Mata, & C/ Po S. Juan de Dios, 2 \\
43206 REUS (TARRAGONA) & ESPLUGAS (BARCELONA) \\
Telef. 600 422875 & Tel. 93 2408000 - Ext. 2374 \\
\hline CARMEN GONZALEZ NOGUERA & ESTEBAN ACOSTA \\
Hospital Insular de Gran Canaria & Hospital de Día- \\
Avda. Marítima del Sur, sn. & C/ Rafael Villa, 65 \\
35016 - LAS PALMAS DE GRANCANARIA & MADRID \\
Tel.928 444291 -99-92 - Fax:928 430032 & \\
Partic. 928 430032 & \\
\hline & \\
\hline
\end{tabular}




\begin{tabular}{|l|l|}
\hline RUBEN ESTANDIA GALAN & MARTA PERAL \\
Centro de Educación Especial «Lupasco» & C/ Campoamor, 3 2 ${ }^{\circ}$ I \\
C/ Lealtad, 3 - ${ }^{\circ}$ Izqda. & 28004 - MADRID \\
Tel 942229799 (Centro) & \\
942 225388 (Consulta) & \\
E-mail: estandia@correo.cop.es & \\
\hline ROSER PEREZ SIMO & CENTRO TERAPEUTICO BELLAIRE \\
Jefe Serv. Psiquiatría & Ramon Llull, 29 - 31 \\
Hospital S. Juan de Dios & 08193 - BELLATERRA \\
C/ S. Juan de Dios, 2 & \\
ESPLUGUES (BARCELONA) & \\
Tel. 93 2804000 Ext. 2361 & \\
Part. 93 4309672 & \\
\hline ISABEL LAUDO & \\
Fundació Hospital Sant Pere Claver & MERCE RUEDA \\
C/ Vila-Vila, 16 & Fundació Hospital Sant Pere Claver \\
BARCELONA & C/ Vila-Vila, 16 \\
Tel.: 93 4423000 & BARCELONA \\
\hline Ma JOSE LAVERNIA PRADES & Tel.: 93 4423000 \\
Hospital de Día & NEUS MOR LLOP \\
Rovira roure, 44 & Hospital de Día \\
LLEIDA & Rovira roure, 44 \\
Tel.: 973 727219 & LLEIDA \\
\hline CRISTINA MOLINS & Tel.: 973 727219 \\
Unidad de Salud Mental de Fuenlabrada & ANA-Ma JIMÉNEZ PASCUAL \\
Leganés, 59 - Posterior & C/ Alto S. Mental Infanto-Juvenil \\
28945 - FUENLABRADA & 13600-ALCAZAR DE SAN JUAN \\
& Tel.-Fax: 926 541750 \\
E-mail: usmas@ @local.jccm.es \\
\hline
\end{tabular}

*Coordinadora del grupo de trabajo de la A.E.N.

Fecha de recepción: Julio de 2000 